\title{
An Existence Theorem for a Nonlocal Global Pandemic Model for Insect-Borne Diseases
}

\author{
John R. Cannon' and Daniel J. Galiffa ${ }^{2}$ \\ ${ }^{1}$ Mathematics Department, University of Central Florida, Orlando, FL 32816, USA \\ ${ }^{2}$ Department of Mathematics, Penn State Erie, The Behrend College, Erie, PA 16563, USA \\ Correspondence should be addressed to Daniel J. Galiffa; djg34@psu.edu \\ Received 6 May 2014; Revised 14 July 2014; Accepted 15 July 2014; Published 24 July 2014 \\ Academic Editor: Kanishka Perera
}

Copyright (C) 2014 J. R. Cannon and D. J. Galiffa. This is an open access article distributed under the Creative Commons Attribution License, which permits unrestricted use, distribution, and reproduction in any medium, provided the original work is properly cited.

We construct and analyze a nonlocal global pandemic model that comprises a system of two nonlocal integrodifferential equations (functional differential equations) and an ordinary differential equation. This model was constructed by considering a spherical coordinate transformation of a previously established epidemiology model that can be applied to insect-borne diseases, like yellow fever. This transformation amounts to a nonlocal boundary value problem on the unit sphere and therefore can be interpreted as a global pandemic model for insect-borne diseases. We ultimately show that a weak solution to the weak formulation of this model exists using a fixed point argument, which calls upon the construction of a weak formulation and the existence and uniqueness of an auxiliary problem.

\section{Introduction}

In [1], Cannon and Galiffa analyzed the nonlocal model:

$$
\begin{gathered}
-\alpha\left(\int_{0}^{1} u(t) \mathrm{d} t\right) u^{\prime \prime}=f(x), \quad x \in(0,1) \\
u(0)=a, \quad u(1)=b,
\end{gathered}
$$

with $\alpha=\alpha(q)$ being a positive function of $q$ defined over $-\infty<q<\infty, f(x)$ defined over $0 \leq x \leq 1$, and $a$ and $b$ real constants. The analysis contained in [1] included both existence and uniqueness theorems for the analytic solution, $u$, to (1) and a discretization, as well as the construction and implementation of a finite-difference procedure. This model was a special case of [2], which has applications to thermodynamics.

The following homogeneous extension of model (1) was considered in [3]:

$$
\begin{gathered}
-\alpha\left(\int_{0}^{1} u(t) \mathrm{d} t\right) u^{\prime \prime}(x)+[u(x)]^{2 n+1}=0, \quad x \in(0,1), \\
u(0)=a, \quad u(1)=b .
\end{gathered}
$$

As in (1), the existence and uniqueness of the analytic solution, $u$, to (2), as well as a discretization, were established and another finite-difference procedure was developed and executed; however, the analysis was significantly different than in [1].

Upon the completion of [3], the authors realized the potential for additional applications to physical problems via mathematical models consisting of variations of the nonlocal term $\alpha\left(\int_{0}^{1} u(t) d t\right)$. In particular, a model was constructed and analyzed in $[4,5]$ that can be applied to insect-borne diseases, for example, yellow fever, which is as follows:

$$
\begin{array}{r}
u_{t}=u_{x x}-\alpha\left(\int_{a}^{b} w(x, t) \mathrm{d} x\right) u+\gamma w, \\
-\infty<x<\infty, \quad t \leq T, \\
v_{t}=v_{x x}+\alpha\left(\int_{a}^{b} w(x, t) \mathrm{d} x\right) u-\beta v, \\
-\infty<x<\infty, \quad t \leq T, \\
w_{t}=\beta v-\gamma w, \quad-\infty<x<\infty, \quad t \leq T,
\end{array}
$$




$$
\begin{aligned}
& u(x, 0)=f(x), \quad-\infty<x<\infty, \\
& v(x, 0)=0, \quad-\infty<x<\infty, \\
& w(x, 0)=0, \quad-\infty<x<\infty
\end{aligned}
$$

with $\beta$ and $\gamma$ arbitrary positive constants.

We next give an overview of this model, which is based on [5]. Biologically, $\beta$ represents the rate at which mobile infecteds become immobile and $\gamma$ represents the rate at which immobile infecteds recover. The unknowns $u, v$, and $w$, respectively, represent a susceptible class, a mobile infected class, and an immobile infected class. In addition, neither infected class is infective; that is, susceptibles become infected via contact with vectors (disease-spreading agents, e.g., Aedes aegypti mosquitoes), which was not modeled directly. The nonnegative function $\alpha(q), 0 \leq q<\infty$, represents the per capita rate at which susceptibles contract the disease in a disease-free population, which is decreasing with $q$. The argument $\int_{a}^{b} w(x, t) \mathrm{d} x$, representing the number of the population immobilized from the disease, when substituted into $\alpha$ brings about a reduction in the rate of infection via actions taken at large and at the individual level, to reduce the risk of infections. Even more specifically, $\int_{a}^{b} w(x, t) \mathrm{d} x$ counts the number of immobile infecteds within a single fixed interval $[a, b]$ and influences the rate $\alpha(q)$ from a biological standpoint. For example, if all news stories about immobile infecteds concern individuals living only in region $[a, b]$, then susceptibles will take precautions based only on the reported number $\int_{a}^{b} w(x, t) \mathrm{d} x$.

In this paper, we consider an extension of model (3) via a spherical coordinate transformation that results in the following model on the unit sphere $\delta$ :

$$
\begin{aligned}
& u_{t}= \frac{1}{\sin \phi} \frac{\partial}{\partial \phi}\left(\sin \phi \frac{\partial u}{\partial \phi}\right)+\csc ^{2} \phi \frac{\partial^{2} u}{\partial \theta^{2}} \\
&-\alpha\left(\int_{\Omega} w(\phi, \theta, t) \sin \phi \mathrm{d} \phi \mathrm{d} \theta\right) u+\gamma w \\
& \text { on } \mathcal{S} \times(0, T] \\
& v_{t}= \frac{1}{\sin \phi} \frac{\partial}{\partial \phi}\left(\sin \phi \frac{\partial w}{\partial \phi}\right)+\csc ^{2} \phi \frac{\partial^{2} v}{\partial \theta^{2}} \\
&+\alpha\left(\int_{\Omega} w(\phi, \theta, t) \sin \phi \mathrm{d} \phi \mathrm{d} \theta\right) u-\beta v \\
& w_{t}=\beta v(\phi, \theta, t)-\gamma w(\phi, \theta, t), \quad \text { on } \mathcal{S} \times(0, T] \\
& u(\phi, \theta, 0)=f(\phi, \theta) \quad \text { on } \mathcal{S} \\
& v(\phi, \theta, 0)=0 \quad \text { on } \mathcal{S}, \\
& w(\phi, \theta, 0)=0 \quad \text { on } \Omega \subset \mathcal{S} .
\end{aligned}
$$

Physically, the system comprising (4) can be interpreted as a preliminary model for a global pandemic of an insect-borne disease. First, we note that the system of spherical coordinates is the natural and historical coordinate system for the surface of the Earth. Second, we note that diffusion has been used to describe epidemics in the past; compare [6-9] for a few of several such examples. Third, the system proposed herein is preliminary, since we take no account of the oceans, as to do so will require boundary conditions to be assigned to the coasts of the Earth's land masses. Fourth, spherical coordinates come into play for relatively short distances on the Earth's surface. For example, a Cartesian coordinate system $(x, y, z)$ with origin located at the John F. Kennedy Space Center will find the plane $(x, y, 0)$ approximately 20,000 feet over Tampa, FL. Fifth, we have made no attempt to model air travel, which can be handled by the introduction of spatially located sources and sinks. Sixth, we note that mosquitoes have been found alive in shipping containers filled with used tires shipped to the United States from Asia, as well as other locations, which provides a vector for tropical Asian diseases on the American continent (cf. http://www.epa.state.il.us/land/tires/mosquito-borne -illnesses.html, for one such example). Moreover, we also note that passengers entering Australia are sprayed with insecticide disinfectant before they can be allowed to deplane (http://abcnews.go.com/).

Finally, our interest here is to show that a solution exists to (4) in order to enable the study of the possible effect of the death toll of a disease on the dynamics of the epidemic itself. Thus, the system (4), which incorporates a coefficient containing a functional of part of the solution of the system, requires a nonlinear analysis, which we will outline later in this introduction.

Several papers have recently been published regarding the mathematical analysis of pandemics, as well as the construction of mathematical models comprising a system of differential equations applicable to such biological phenomena. Several of these papers influenced our current work and it is therefore worth briefly describing them before we further discuss (4).

For example, in [10] the authors explain how the utilization of antiviral drugs was intended to moderate the severity of a new strain of an influenza pandemic and that the success of these drugs was dependent upon the timely onset of therapy (within 48 hours) after the appearance of clinical symptoms. They discuss further that this requirement can be understood by a compartmental model that examines the density of infected individuals in terms of the time elapsed since the onset of symptoms. Therefore, based on this compartmental model, a system of delay-differential equations, with both discrete and disturbed delays, was constructed and analyzed. This system is as follows:

$$
\begin{gathered}
\frac{d}{d t} S(t)=-\beta S(t) Q(t), \\
\frac{d}{d t} E(t)=\beta S(t) Q(t)-\mu_{E} E(t),
\end{gathered}
$$


for $t \geq 0$, where susceptible and exposed classes are, respectively, denoted by $S$ and $E$, with $\beta$ representing the baseline transmission rate, $1 / \mu_{E}$ representing the incubation period, and $Q(t)$ representing the force of infection yet to be formulated.

As a precaution to a potential $\mathrm{H} 5 \mathrm{~N} 1$ pandemic, the authors in [11] simulate a possible outbreak in Italy by analyzing the structure and the effect of including more specific details like heterogeneities and stochasticity. More specifically, the heterogeneity was with respect to age and spatial distribution, and the stochasticity regulated the epidemic dynamics when the number of the infected population is low. This resulted in the SEIR model:

$$
\begin{gathered}
\frac{d}{d t} S_{i}^{p}=-S_{i}^{p} \sum_{j, q} \beta_{i j}^{p q} \frac{I_{j}^{q}}{N_{j}^{q}}, \quad \frac{d}{d t} I_{i}^{p}=\nu E_{i}^{p}-\gamma I_{i}^{p}, \\
\frac{d}{d t} E_{i}^{p}=S_{i}^{p} \sum_{j, q} \beta_{i j}^{p q} \frac{I_{j}^{q}}{N_{j}^{q}}-v E_{i}^{p}, \quad \frac{d}{d t} R_{i}^{p}=I_{i}^{p},
\end{gathered}
$$

with $i, j=1, \ldots, 12$ and $p, q=1, \ldots, 20$, where $1 / \nu$ and $1 / \gamma$, respectively, represent the mean length of the latency and infectivity periods.

Other fairly recent papers related to the modeling of pandemics are worth mentioning here as well. For example, in [12] genetic algorithms were used in order to determine optimal vaccination strategies for pandemic influenza. Also, in [13] in order to analyze recurrent outbreaks of the avian H5N1 influenza virus in Asia, which constitute a global pandemic threat, the authors analyzed the great influenza pandemic of 1918 in Geneva, Switzerland, which is also referred to as the "Spanish flu." Lastly, [14] shows how previous constraints regarding continuous space contact models describing a class of multitype epidemics can be removed in order to construct a general proof of the pandemic bonds for the multitype epidemic on the lattice $\mathbb{Z}^{N}$. For further interesting results related to recent studies of pandemics consider [15-17].

Our current model is quite different than the aforementioned ones, since it comprises two nonlocal integrodifferential equations, also often referred to as functional differential equations, and an ordinary differential equation. Moreover, in order to establish the existence of the weak solution to the weak formulation of (4), an interesting Hilbert space is constructed, as established in Section 3. In addition, several facets of the analysis needed in the study of Laplace's equation on the unit sphere are used throughout. We also emphasize that, to the very best of our knowledge, there are currently no published results related to nonlocal global pandemic models.

This paper is organized as follows. In Section 2, we derive a weak formulation of (4). Based on this analysis, an auxiliary problem is constructed in Section 3, from which we show that its solution exists and is unique. In Section 4, we prove the existence of a solution to the weak formulation of model (4), using the analysis of Section 3. We conclude the paper with Section 5, wherein we briefly discuss future considerations.

\section{A Weak Formulation}

We begin by multiplying each equation in (4) by $\sin \phi$, resulting in

$$
\begin{aligned}
u_{t} \sin \phi= & \frac{\partial}{\partial \phi}\left(\sin \phi \frac{\partial u}{\partial \phi}\right)+\csc \phi \frac{\partial^{2} u}{\partial \theta^{2}} \\
& -\alpha\left(\int_{\Omega} w(\phi, \theta, t) \sin \phi \mathrm{d} \phi \mathrm{d} \theta\right) u \sin \phi \\
& +\gamma w \sin \phi, \quad \text { on } \mathcal{S} \times(0, T] \\
v_{t} \sin \phi= & \frac{\partial}{\partial \phi}\left(\sin \phi \frac{\partial w}{\partial \phi}\right)+\csc \phi \frac{\partial^{2} v}{\partial \theta^{2}} \\
& +\alpha\left(\int_{\Omega} w(\phi, \theta, t) \sin \phi \mathrm{d} \phi \mathrm{d} \theta\right) u \sin \phi \\
& -\beta v \sin \phi, \quad \text { on } \mathcal{S} \times(0, T], \\
w_{t}=\beta v(\phi, \theta, t)-\gamma w(\phi, \theta, t), \quad \text { on } \mathcal{S} \times(0, T] & \\
u(\phi, \theta, 0)= & f(\phi, \theta) \quad \text { on } \mathcal{S}, \quad v(\phi, \theta, 0)=0 \quad \text { on } \mathcal{S}, \\
& w(\phi, \theta, 0)=0 \quad \text { on } \Omega \subset \mathcal{S} .
\end{aligned}
$$

Moreover, $u, v$, and $w$, as well as $\partial u / \partial \theta, \partial v / \partial \theta$, and $\partial w / \partial \theta$, are all continuous across the data line, and all of the derivatives with respect to $\phi$ of $u, v$, and $w$ exist for every direction $\theta$ when $\phi=0, \pi$.

In considering a weak solution to (7)-(9), we need to establish appropriate test functions. Consider $g(\phi, \theta, t) \in C^{1}$ in $\phi, \theta$, and $t$ and let $g$ and $g_{\theta}$ be continuous across the data line. Now multiply (7) by $g(\phi, \theta, t)$ and let $g(\phi, \theta, T)=0$; then

$$
\begin{aligned}
\int_{0}^{T} \int_{-\pi}^{\pi} \int_{0}^{\pi} g u_{t} \sin \phi \mathrm{d} \phi \mathrm{d} \theta \mathrm{d} t \\
=-\int_{-\pi}^{\pi} \int_{0}^{\pi} g(\phi, \theta, 0) f(\phi, \theta) \sin \phi \mathrm{d} \phi \mathrm{d} \theta \\
\quad-\int_{0}^{T} \int_{-\pi}^{\pi} \int_{0}^{\pi} u g_{t} \sin \phi \mathrm{d} \phi \mathrm{d} \theta \mathrm{d} t .
\end{aligned}
$$

The next term in (7) yields

$$
\begin{aligned}
\int_{0}^{T} \int_{-\pi}^{\pi} \int_{0}^{\pi} g \frac{\partial}{\partial \phi}\left(\sin \phi u_{\phi}\right) \mathrm{d} \phi \mathrm{d} \theta \mathrm{d} t \\
\quad=\left.\int_{0}^{T} \int_{-\pi}^{\pi} g u_{\phi} \sin \phi\right|_{\phi=0} ^{\phi=\pi} \mathrm{d} \theta \mathrm{d} t \\
\quad-\int_{0}^{T} \int_{-\pi}^{\pi} \int_{0}^{\pi} g u_{\phi} \sin \phi \mathrm{d} \phi \mathrm{d} \theta \mathrm{d} t .
\end{aligned}
$$


We also have

$$
\begin{aligned}
\int_{0}^{T} \int_{0}^{\pi} \int_{-\pi}^{\pi} \csc \phi g u_{\theta \theta} \mathrm{d} \theta_{\phi} \mathrm{d} \phi \mathrm{d} t \\
\quad=\left.\int_{0}^{T} \int_{0}^{\pi} \csc \phi g u_{\theta}\right|_{\theta=-\pi} ^{\theta=\pi} \mathrm{d} \phi \mathrm{d} t \\
\quad-\int_{0}^{T} \int_{0}^{\pi} \int_{-\pi}^{\pi} \csc \phi g_{\theta} u_{\theta} \mathrm{d} \theta \mathrm{d} \phi \mathrm{d} t
\end{aligned}
$$

Rearranging terms we obtain

$$
\begin{aligned}
-\int_{0}^{T} \int_{-\pi}^{\pi} \int_{0}^{\pi} u g_{t} \sin \phi \mathrm{d} \phi \mathrm{d} \theta \mathrm{d} t \\
\quad-\int_{0}^{T} \int_{-\pi}^{\pi} \int_{0}^{\pi} g_{\phi} u_{\phi} \sin \phi \mathrm{d} \phi \mathrm{d} \theta \mathrm{d} t \\
\quad+\int_{0}^{T} \int_{-\pi}^{\pi} \int_{0}^{\pi} \csc \phi g_{\theta} u_{\theta} \mathrm{d} \phi \mathrm{d} \theta \mathrm{d} t \\
=\int_{-\pi}^{\pi} \int_{0}^{\pi} g(\phi, \theta, 0) f(\phi, \theta) \sin \phi \mathrm{d} \phi \mathrm{d} \theta \\
\quad-\int_{0}^{T} \int_{-\pi}^{\pi} \int_{0}^{\pi} \alpha\left(\int_{\Omega} w \sin \phi \mathrm{d} \phi \mathrm{d} \theta\right) g_{\phi} u \sin \phi \mathrm{d} \phi \mathrm{d} \theta \mathrm{d} t \\
\quad+\gamma \int_{0}^{T} \int_{-\pi}^{\pi} \int_{0}^{\pi} g_{\phi} w \sin \phi \mathrm{d} \phi \mathrm{d} \theta,
\end{aligned}
$$

for all $g \in C^{1}\left(Q_{T}\right)$ and $Q_{T}=\{(\phi, \theta, t) \mid 0 \leq \phi \leq \pi,-\pi \leq \theta \leq$ $\pi, 0<t \leq T\}$.

Now let $D_{t}:=\{(\phi, \theta, t) \mid 0 \leq \phi \leq \pi,-\pi \leq \theta \leq \pi\}$. For ease of notation we assign the following:

$$
\begin{gathered}
\int_{Q_{T}} u g_{t} \sin \phi \mathrm{d} Q_{T}:=\int_{0}^{T} \int_{-\pi}^{\pi} \int_{0}^{\pi} u g_{t} \sin \phi \mathrm{d} \phi \mathrm{d} \theta \mathrm{d} t, \\
\int_{D_{t}} u g_{t} \sin \phi \mathrm{d} D_{t}:=\int_{-\pi}^{\pi} \int_{0}^{\pi} u g_{t} \sin \phi \mathrm{d} \phi \mathrm{d} \theta, \\
\int_{\Omega} w \mathrm{~d} \Omega:=\int_{\Omega} \int w(\phi, \theta, t) \sin \phi \mathrm{d} \phi \mathrm{d} \theta, \quad \text { where } \Omega \subseteq \mathcal{S} .
\end{gathered}
$$

We can then write the weak form of (7) as follows:

$$
\begin{aligned}
& -\int_{Q_{T}} u g_{t} \sin \phi \mathrm{d} Q_{T}-\int_{Q_{T}} u_{\phi} g_{\phi} \sin \phi \mathrm{d} Q_{T} \\
& \quad+\int_{Q_{T}} u_{\theta} g_{\theta} \csc \phi \mathrm{d} Q_{T} \\
& =\int_{D_{0}} g f \sin \phi \mathrm{d} D_{0}-\int_{Q_{T}} \alpha\left(\int_{\Omega} w \mathrm{~d} \Omega\right) u g \sin \phi \mathrm{d} Q_{T} \\
& \quad+\gamma \int_{Q_{T}} g w \sin \phi \mathrm{d} Q_{T},
\end{aligned}
$$

for all $g \in C^{1}\left(Q_{T}\right)$ and $g(\phi, \theta, T)=0$, or equivalently for all $g \in W^{1,1}$, which is the closure of $C^{1}\left(Q_{T}\right)$ with $g(\phi, \theta, T)=0$ in the norm

$$
\begin{aligned}
\|g\|_{W^{1,1}}^{2}:= & \int_{Q_{T}} g^{2} \sin \phi \mathrm{d} Q_{T}+\int_{Q_{T}} g_{\theta}^{2} \sin \phi \mathrm{d} Q_{T} \\
& +\int_{Q_{T}} g_{\theta}^{2} \csc \phi \mathrm{d}_{T}+\int_{Q_{T}} g_{t}^{2} \sin \phi \mathrm{d} Q_{T} .
\end{aligned}
$$

For the weak form of (8) we have

$$
\begin{gathered}
-\int_{\mathrm{Q}_{T}} v g_{t} \sin \phi \mathrm{d} Q_{T}+\int_{\mathrm{Q}_{T}} v_{\phi} g_{\phi} \sin \phi \mathrm{d} Q_{T} \\
\quad+\int_{Q_{T}} v_{\theta} g_{\theta} \csc \phi \mathrm{d}_{T} \\
=\int_{Q_{T}} \alpha\left(\int_{\Omega} w \mathrm{~d} \Omega\right) u g \sin \phi \mathrm{d} Q_{T} \\
\quad-\beta \int_{Q_{T}} v g \sin \phi \mathrm{d} Q_{T}, \quad \forall g \in W^{1,1} .
\end{gathered}
$$

On the other hand, the weak form of (9) is the pointwise integral representation for almost all $(\phi, \theta, t) \in Q_{T}$, which is

$$
w=\beta \int_{0}^{t} v e^{-\gamma(t-\tau)} \mathrm{d} \tau .
$$

The integral representation (19) of $w$ is a nonlinear functional equation for $w$ as $\int_{\Omega} w \mathrm{~d} \Omega$ occurs in the function $\alpha$ in both (16) and (18) for $u$ and $v$.

\section{The Auxiliary Problem}

We replace $\int_{\Omega} w \mathrm{~d} \Omega$ in the function $\alpha$ in (16) and (18) with the continuous function $q(t)$ to yield the auxiliary problem of finding $u(\phi, \theta, t ; q), v(\phi, \theta, t ; q)$, and $w(\phi, \theta, t ; q)$ satisfying

$$
\begin{array}{rl}
-\int_{\mathrm{Q}_{T}} & u g_{t} \sin \phi \mathrm{d} Q_{T}+\int_{Q_{T}} u_{\phi} g_{\phi} \sin \phi \mathrm{d} Q_{T} \\
& +\int_{Q_{T}} u_{\theta} g_{\theta} \csc \phi \mathrm{d} Q_{T} \\
= & \int_{D_{0}} g f \sin \phi \mathrm{d} D_{0}-\int_{Q_{T}} \alpha(q(t)) u g \sin \phi \mathrm{d} Q_{T} \\
& +\gamma \int_{Q_{T}} g w \sin \phi \mathrm{d} Q_{T}, \quad \forall g \in W^{1,1},
\end{array}
$$




$$
\begin{gathered}
-\int_{\mathrm{Q}_{T}} v g_{t} \sin \phi \mathrm{d} Q_{T}+\int_{\mathrm{Q}_{T}} v_{\phi} g_{\phi} \sin \phi \mathrm{d} Q_{T} \\
\quad+\int_{\mathrm{Q}_{T}} v_{\theta} g_{\theta} \csc \phi \mathrm{d} Q_{T} \\
=\int_{Q_{T}} \alpha(q(t)) u g \sin \phi d Q_{T} \\
-\beta \int_{Q_{T}} v g \sin \phi \mathrm{d} Q_{T}, \quad \forall g \in W^{1,1}, \\
w(\phi, \theta, t ; q)=\beta \int_{0}^{t} v(\phi, \theta, \tau ; q) e^{-\gamma(t-\tau)} \mathrm{d} \tau
\end{gathered}
$$

for almost all $(\phi, \theta, t) \in Q_{T}$. It should also be noted that we have already assumed that $g$ is periodic in $\theta$. Also, we can extend $g$ as an odd function in $\phi$ with $-\pi \leq \phi \leq 0$ and regard it as periodic in $\phi$. Therefore, we can utilize the functions $\sin n \phi \sin m \theta$ and $\sin n \phi \cos m \theta$ in order to generate an indexed set of functions $h_{k}(\phi, \theta)$ by first counting the functions $\sin n \phi \cos m \theta$ according to an orderly method of counting the ordered pairs $(n, m)$ for $n=1,2, \ldots$ and $m=$ $0,1,2, \ldots$ in the closure of the $x-y$ plane via the diagonal pattern

$$
\begin{aligned}
(1,0) & \longrightarrow(1,1) \longrightarrow(2,0) \longrightarrow(3,0) \longrightarrow(2,1) \longrightarrow(1,2) \\
& \longrightarrow(1,3) \longrightarrow(2,2) \longrightarrow(3,1) \longrightarrow(4,0) \longrightarrow(5,0) \\
& \longrightarrow(4,1) \longrightarrow(3,2) \longrightarrow(2,3) \longrightarrow(1,4) \longrightarrow(1,5) \\
& \longrightarrow(2,4) \cdots
\end{aligned}
$$

Next, take the number index assigned to each pair $(n, m)$ and double it, yielding the $h_{k}$-terms for even integers. Then, for the odd integers, as we did above, count the functions $\sin n \phi \cos m \theta$ according to an orderly method of counting the ordered pairs $(n, m)$ for $n=1,2, \ldots$ and $m=1,2, \ldots$ in the closure of the $x-y$ plane via the diagonal pattern

$$
\begin{aligned}
(1,1) & \longrightarrow(1,2) \longrightarrow(2,1) \longrightarrow(3,1) \longrightarrow(2,2) \\
& \longrightarrow(1,3) \longrightarrow(1,4) \longrightarrow(2,3) \longrightarrow(3,2) \\
& \longrightarrow(4,1) \longrightarrow(5,1) \longrightarrow(4,2) \longrightarrow(3,3) \\
& \longrightarrow(2,4) \longrightarrow(1,5) \longrightarrow(1,6) \longrightarrow(2,5) \cdots
\end{aligned}
$$

So, we have a list of $h_{k}(\phi, \theta)$ for $k=1,2, \ldots$. The first six of such are as follows:

$$
\begin{array}{ll}
h_{1}=\sin (\phi) \sin (\theta), & h_{2}=\sin (\phi), \\
h_{3}=\sin (\phi) \sin (2 \theta), & h_{4}=\sin (\phi) \cos (\theta), \\
h_{5}=\sin (2 \phi) \sin (\theta), & h_{6}=\sin (2 \phi), \ldots
\end{array}
$$

Since the integrals involving the $h_{k}^{2}$-term, given by

$$
\begin{aligned}
& \int_{-\pi}^{\pi} \int_{0}^{\pi} \sin ^{2} n \phi \cos ^{2} m \theta \sin \phi \mathrm{d} \phi \mathrm{d} \theta, \\
& \int_{-\pi}^{\pi} \int_{0}^{\pi} \sin ^{2} n \phi \sin ^{2} m \theta \sin \phi \mathrm{d} \phi \mathrm{d} \theta,
\end{aligned}
$$

converge, and the integrals involving the $\partial h_{k}^{2} / \partial \phi$-term, which are

$$
\begin{aligned}
& \int_{-\pi}^{\pi} \int_{0}^{\pi} n^{2} \cos ^{2} n \phi \cos ^{2} m \theta \sin \phi \mathrm{d} \phi \mathrm{d} \theta, \\
& \int_{-\pi}^{\pi} \int_{0}^{\pi} n^{2} \cos ^{2} n \phi \sin ^{2} m \theta \sin \phi \mathrm{d} \phi \mathrm{d} \theta,
\end{aligned}
$$

also converge, we then consider the integrals involving the $\partial h_{k}^{2} / \partial \theta$-term below:

$$
\begin{aligned}
& \int_{-\pi}^{\pi} \int_{0}^{\pi} m^{2} \csc \phi \sin ^{2} n \phi \sin ^{2} m \theta d \phi d \theta, \\
& \int_{-\pi}^{\pi} \int_{0}^{\pi} m^{2} \csc \phi \sin ^{2} n \phi \cos ^{2} m \theta d \phi d \theta
\end{aligned}
$$

and remark that

$$
\int_{0}^{\pi} m^{2} \csc \phi \sin ^{2} n \phi d \phi<\frac{n^{2} m^{2} \pi^{4}}{8} .
$$

Hence,

$$
\begin{gathered}
\int_{-\pi}^{\pi} \int_{0}^{\pi} h_{k}^{2} \sin \phi \mathrm{d} \phi \mathrm{d} \theta+\int_{-\pi}^{\pi} \int_{0}^{\pi}\left(\frac{\partial h_{k}}{\partial \phi}\right)^{2} \sin \phi \mathrm{d} \phi \mathrm{d} \theta \\
+\int_{-\pi}^{\pi} \int_{0}^{\pi}\left(\frac{\partial h_{k}}{\partial \theta}\right)^{2} \csc \phi \mathrm{d} \phi \mathrm{d} \theta<\infty,
\end{gathered}
$$

for $k=1,2, \ldots$ Thus, via the Gram-Schmidt process, we can generate a countable orthonormal basis for the Hilbert space $\mathscr{L}^{2}\left(D_{0}\right)$ with the inner product

$$
\begin{aligned}
\langle f(\phi, \theta), g(\phi, \theta)\rangle_{D_{0}}= & \int_{D_{0}} f g \sin \phi \mathrm{d} D_{0} \\
& +\int_{D_{0}} \frac{\partial f}{\partial \phi} \frac{\partial g}{\partial \phi} \sin \phi \mathrm{d} D_{0} \\
& +\int_{D_{0}} \frac{\partial f}{\partial \theta} \frac{\partial g}{\partial \theta} \csc \phi \mathrm{d} D_{0},
\end{aligned}
$$

where $D_{0}=\{(\phi, \theta) \mid 0 \leq \phi \leq \pi,-\pi \leq \theta \leq \pi\}$. Henceforth, we denote the orthonormal basis formed from $h_{k}(\phi, \theta)$ via the Gram-Schmidt process by $\left\{h_{k}(\phi, \theta)\right\}$.

Now, define

$$
\begin{aligned}
& u_{N}(\phi, \theta, t)=\sum_{k=1}^{N} a_{k}^{N} h_{k}(\phi, \theta), \\
& v_{N}(\phi, \theta, t)=\sum_{k=1}^{N} b_{k}^{N} h_{k}(\phi, \theta), \\
& w_{N}(\phi, \theta, t)=\sum_{k=1}^{N} c_{k}^{N} h_{k}(\phi, \theta) .
\end{aligned}
$$


From there, we construct a system of ODEs as follows:

$$
\begin{aligned}
& \int_{D_{0}} u_{n} h_{i} \sin \phi \mathrm{d} D_{0}+\int_{D_{0}} \frac{\partial u_{N}}{\partial \phi} \frac{\partial h_{i}}{\partial \phi} \sin \phi \mathrm{d} D_{0} \\
& \quad+\int_{D_{0}} \frac{\partial u_{N}}{\partial \theta} \frac{\partial h_{i}}{\partial \theta} \csc \phi \mathrm{d} D_{0} \\
& =-\int_{D_{0}} \alpha(q(t)) u_{N} h_{i} \sin \phi \mathrm{d} D_{0}+\gamma \int_{D_{0}} w_{N} h_{i} \sin \phi \mathrm{d} D_{0} \\
& \int_{D_{0}} v_{n} h_{i} \sin \phi \mathrm{d} D_{0}+\int_{D_{0}} \frac{\partial v_{N}}{\partial \phi} \frac{\partial h_{i}}{\partial \phi} \sin \phi \mathrm{d} D_{0} \\
& \quad+\int_{D_{0}} \frac{\partial v_{N}}{\partial \theta} \frac{\partial h_{i}}{\partial \theta} \csc \phi \mathrm{d} D_{0} \\
& =\int_{D_{0}} \alpha(q(t)) u_{N} h_{i} \sin \phi \mathrm{d} D_{0}-\beta \int_{D_{0}} v_{N} h_{i} \sin \phi \mathrm{d} D_{0}, \\
& \int_{D_{0}} w_{n} h_{i} \sin \phi \mathrm{d} D_{0}=\beta \int_{D_{0}} v_{N} h_{i} \sin \phi \mathrm{d} D_{0} \\
& \quad-\gamma \int_{D_{0}} w_{N} h_{i} \sin \phi \mathrm{d} D_{0}, \\
& a_{i}^{N}(0)=\left\langle f, h_{i}\right\rangle_{D_{0}}, \quad b_{i}^{N}(0)=c_{i}^{N}(0)=0, i=1,2, \ldots, N .
\end{aligned}
$$

The equations above become

$$
\begin{gathered}
\frac{d a_{i}^{N}(t)}{d t}+\sum_{l=1}^{N} A_{i, l}^{N} a_{l}^{N}(t)=-\alpha(q(t)) a_{i}^{N}(t)+\gamma c_{i}^{N}(t), \\
\frac{d b_{i}^{N}(t)}{d t}+\sum_{l=1}^{N} B_{i, l}^{N} b_{l}^{N}(t)=\alpha(q(t)) a_{i}^{N}(t)-\beta b_{i}^{N}(t), \\
\frac{d c_{i}^{N}(t)}{d t}=\beta b_{i}^{N}(t)-\gamma c_{i}^{N}(t), \\
a_{i}^{N}(0)=\left\langle f, h_{i}\right\rangle_{D_{0}}, \quad b_{i}^{N}(0)=c_{i}^{N}(0)=0 \\
i=1,2, \ldots, N .
\end{gathered}
$$

As the above equations are linear, for each positive integer $N$ there exists a solution on $[0, T]$ since $\alpha(q(t))$ is bounded for $q(t) \geq 0$. Hence, for each $N, u_{N}, v_{N}$, and $w_{N}$ are determined uniquely. We note that

$$
c_{i}^{N}(t)=\beta \int_{0}^{t} b_{i}^{N}(\tau) e^{\gamma(t-\tau)} \mathrm{d} \tau
$$

Upon multiplying each of the equations in the system (32) by the coefficient corresponding to the $t$-derivative in the equation and summing each equation over $i=1, \ldots, N$, we achieve

$$
\begin{aligned}
& \left\langle\frac{d u_{N}}{d t}, u_{N}\right\rangle_{D_{0}}+\int_{D_{0}}\left(\frac{\partial u_{N}}{\partial \phi}\right)^{2} \sin \phi \mathrm{d} D_{0} \\
& +\int_{D_{0}}\left(\frac{\partial u_{N}}{\partial \theta}\right)^{2} \csc \phi \mathrm{d} D_{0} \\
& =-\int_{D_{0}} \alpha(q(t)) u_{N}^{2} \sin \phi \mathrm{d} D_{0} \\
& +\gamma \int_{D_{0}} w_{N} u_{N} \sin \phi \mathrm{d} D_{0}, \\
& \left\langle\frac{d v_{N}}{d t}, v_{N}\right\rangle_{D_{0}}+\int_{D_{0}}\left(\frac{\partial v_{N}}{\partial \phi}\right)^{2} \sin \phi \mathrm{d} D_{0} \\
& +\int_{D_{0}}\left(\frac{\partial v_{N}}{\partial \theta}\right)^{2} \csc \phi \mathrm{d} D_{0} \\
& =\int_{D_{0}} \alpha(q(t)) u_{N} v_{N} \sin \phi \mathrm{d} D_{0} \\
& +\beta \int_{D_{0}} v_{N}^{2} \sin \phi \mathrm{d} D_{0}, \\
& \left\langle\frac{d w_{N}}{d t}, w_{N}\right\rangle_{D_{0}}=\beta \int_{D_{0}} v_{N} w_{N} \sin \phi \mathrm{d} D_{0} \\
& -\gamma \int_{D_{0}} w_{N}^{2} \sin \phi \mathrm{d} D_{0}
\end{aligned}
$$

and integrating each of the above equations from 0 to $t$ we obtain

$$
\begin{aligned}
& \frac{1}{2}\left\|u_{N}(\phi, \theta, t)\right\|_{D_{0}}^{2}+\int_{Q_{t}}\left(\frac{\partial u_{N}}{\partial \phi}\right)^{2} \sin \phi \mathrm{d} Q_{t} \\
& \quad+\int_{Q_{t}}\left(\frac{\partial u_{N}}{\partial \theta}\right)^{2} \csc \phi \mathrm{d}_{t} \\
& =\frac{1}{2}\left\|u_{N}(\phi, \theta, 0)\right\|_{D_{0}}^{2}-\int_{Q_{t}} \alpha(q(t)) u_{N}^{2} \sin \phi \mathrm{d} Q_{t} \\
& \quad+\gamma \int_{Q_{t}} w_{N} u_{N} \sin \phi \mathrm{d} Q_{t} \\
& \frac{1}{2}\left\|v_{N}(\phi, \theta, t)\right\|_{D_{0}}^{2}+\int_{Q_{t}}\left(\frac{\partial v_{N}}{\partial \phi}\right)^{2} \sin \phi \mathrm{d} Q_{t} \\
& \quad+\int_{Q_{t}}\left(\frac{\partial v_{N}}{\partial \theta}\right)^{2} \csc \phi \mathrm{d} Q_{t} \\
& =\int_{Q_{t}} \alpha(q(t)) u_{N} v_{N} \sin \phi \mathrm{d} Q_{t}-\beta \int_{Q_{t}} v_{N}^{2} \sin \phi \mathrm{d} Q_{t} \\
& \frac{1}{2}\left\|w_{N}(\phi, \theta, t)\right\|_{D_{0}}^{2}=\beta \int_{Q_{t}} v_{N} w_{N} \sin \phi \mathrm{d} Q_{t} \\
& -\gamma \int_{Q_{t}} w_{N}^{2} \sin \phi \mathrm{d} Q_{t} .
\end{aligned}
$$


Multiplying the above equations by 2, utilizing the inequality $2 a b \leq a^{2}+b^{2}$ on the right-hand sides of each equation, adding the inequalities, and dropping the $Q_{t}$ integrals on the lefthand side of the resulting inequalities, we have

$$
F(t) \leq\|f\|_{D_{0}}^{2}+C_{1} \int_{0}^{t} F(\tau) \mathrm{d} \tau,
$$

where

$$
\begin{gathered}
F(t):=\left\|u_{N}(\phi, \theta, t)\right\|_{D_{0}}^{2}+\left\|v_{N}(\phi, \theta, t)\right\|_{D_{0}}^{2} \\
+\left\|w_{N}(\phi, \theta, t)\right\|_{D_{0}}^{2}, \\
C_{1}=3\|\alpha\|_{\infty}+3 \beta+2 \gamma+1
\end{gathered}
$$

and $\left\|u_{N}(\phi, \theta, 0)\right\|_{D_{0}}^{2} \leq\|f\|_{D_{0}}^{2}$.

Then, by Gronwall's lemma we see that

$$
\begin{gathered}
y^{\prime} \leq\|f\|_{D_{0}}^{2}+C_{1} y \\
\frac{d}{d t}\left(y e^{-C_{1} t}\right) \leq\|f\|_{D_{0}}^{2} e^{-C_{1} t} \\
y(t) \leq \frac{\|f\|_{D_{0}}^{2}}{C_{1}}\left[e^{C_{1} t}-1\right] .
\end{gathered}
$$

Therefore,

$$
F(t) \leq\|f\|_{D_{0}}^{2} e^{C_{1} t} .
$$

In addition, we have

$$
\begin{aligned}
F(t)+ & 2 \int_{Q_{t}}\left(\frac{\partial u_{N}}{\partial \phi}\right)^{2} \sin \phi \mathrm{d} Q_{t}+2 \int_{Q_{t}}\left(\frac{\partial u_{N}}{\partial \theta}\right)^{2} \csc \phi \mathrm{d} Q_{t} \\
& +2 \int_{Q_{t}}\left(\frac{\partial v_{N}}{\partial \phi}\right)^{2} \sin \phi \mathrm{d}_{t}+2 \int_{Q_{t}}\left(\frac{\partial v_{N}}{\partial \theta}\right)^{2} \csc \phi \mathrm{d}_{t} \\
& \leq\|f\|_{D_{0}}^{2}+C_{1} \int_{0}^{t} F(\tau) d \tau
\end{aligned}
$$

and we observe that the entire left-hand side of the above inequality is also bounded by $\|f\|_{D_{0}}^{2} e^{C_{1} t}$ for each $t \in[0, T]$ and so is each individual term on the left-hand side.

From there, we see that

$$
\begin{gathered}
\int_{Q_{T}} u_{N}^{2} \sin \phi \mathrm{d} Q_{T}, \quad \int_{Q_{T}} v_{N}^{2} \sin \phi \mathrm{d} Q_{T}, \\
\int_{Q_{T}} w_{N}^{2} \sin \phi \mathrm{d} Q_{T}
\end{gathered}
$$

are all bounded uniformly with respect to $N$. Likewise, $u_{N}$ and $v_{N}$ are also uniformly bounded in the Hilbert space $\mathscr{V}$ with inner product

$$
\begin{aligned}
\langle h, g\rangle_{\mathscr{V}}= & \int_{\mathrm{Q}_{T}} h g \sin \phi \mathrm{d} Q_{T}+\int_{\mathrm{Q}_{T}} h_{\phi} g_{\phi} \sin \phi \mathrm{d} Q_{T} \\
& +\int_{Q_{T}} h_{\theta} g_{\theta} \csc \phi \mathrm{d} Q_{T},
\end{aligned}
$$

where $h_{\phi}, g_{\phi}, h_{\theta}$, and $g_{\theta}$ are the first partial derivatives of $h$ and $g$, with respect to the subscripted variables.
Next, we take (33) and multiply it by $h_{i}(\phi, \theta)$ and sum with $i$ from 1 to $N$ and obtain

$$
\begin{array}{r}
w_{N}(\phi, \theta, t)=\beta \int_{0}^{t} v_{N}(\phi, \theta, \tau) e^{-\gamma(t-\tau)} \mathrm{d} \tau, \\
\forall(\phi, \theta, t) \in Q_{T} .
\end{array}
$$

Thus, by a Cantor diagonalization process, there exist sequences $\left\{u_{N}\right\},\left\{v_{N}\right\}$, and $\left\{w_{N}\right\}$, which converge weakly in $\mathscr{L}^{2}\left(Q_{T}\right)$ with $\left\{u_{N}\right\}$ and $\left\{v_{N}\right\}$ also converging weakly in $\mathscr{V}$. Let $u, v$, and $w$ denote the weak limits and let

$$
g_{m}(\phi, \theta, t)=\sum_{i=1}^{m} e_{i}(t) h_{i}(\phi, \theta)
$$

denote an approximation in $W_{2}^{1,1}\left(Q_{T}\right)$ to $g(\phi, \theta, t) \in C_{1}\left(Q_{T}\right)$ with $e_{i}(T)=0$ for all $i=1,2, \ldots$; that is, $g_{m} \rightarrow g$ strongly in $W_{2}^{1,1}\left(Q_{T}\right)$ and $g_{m}(\phi, \theta, T)=g(\phi, \theta, T)=0$. Taking the system of ODEs and multiplying each by $e_{i}(t)$ and summing each block of equations with respect to $i$ transform the system as follows:

$$
\begin{gathered}
\left\langle\frac{\partial u_{N}}{\partial t}, g_{m}\right\rangle_{D_{0}}+\int_{D_{0}} \frac{\partial u_{N}}{\partial \phi} \frac{\partial u_{N}}{\partial \phi} g_{m} \sin \phi \mathrm{d} D_{0} \\
+\int_{D_{0}} \frac{\partial u_{N}}{\partial \theta} \frac{\partial u_{N}}{\partial \theta} g_{m} \csc \phi \mathrm{d} D_{0} \\
=\int_{D_{0}} \alpha(q(t)) u_{N} g_{m} \sin \phi \mathrm{d} D_{0} \\
+\gamma \int_{D_{0}} w_{N} g_{m} \sin \phi \mathrm{d} D_{0}, \\
\left\langle\frac{\partial v_{N}}{\partial t}, g_{m}\right\rangle_{D_{0}}+\int_{D_{0}} \frac{\partial v_{N}}{\partial \phi} \frac{\partial v_{N}}{\partial \phi} g_{m} \sin \phi \mathrm{d} D_{0} \\
\quad+\int_{D_{0}} \frac{\partial v_{N}}{\partial \theta} \frac{\partial v_{N}}{\partial \theta} g_{m} \csc \phi \mathrm{d} D_{0} \\
=\int_{D_{0}} \alpha(q(t)) u_{N} g_{m} \sin \phi \mathrm{d} D_{0} \\
-\beta \int_{D_{0}} v_{N} g_{m} \sin \phi \mathrm{d} D_{0}, \\
\int_{Q_{T}}\left(w_{N}-\beta \int_{0}^{t} v_{N} e^{-\gamma(t-\tau)} \mathrm{d} \tau\right) g_{m} \mathrm{~d} Q_{T}=0 .
\end{gathered}
$$

Integrating with respect to $t$ from 0 to $T$ and performing integration by parts on the first term in each equation, we observe that $u_{N}, v_{N}$, and $w_{N}$ satisfy the weak formulation for $g_{m}$. Then holding $g_{m}$ fixed and letting $N \rightarrow \infty$ we see that the weak limits $u, v$, and $w$ satisfy the original system (20) for each $m$. As $g_{m} \rightarrow g \in C^{1}\left(Q_{t}\right)$ in the norm of $W_{2}^{1,1}\left(Q_{t}\right)$, then $u, v$, and $w$ satisfy the weak formulation for all $g \in C^{1}\left(Q_{t}\right) \cap W_{2}^{1,1}$ and $g(\phi, \theta, T)=0$. Thus, as $g$ is dense in $W_{2}^{1,1}$ the weak limits $u, v$, and $w$ together form a weak solution to the auxiliary problem (20). 
Upon considering the energy inequality for parabolic equations (cf. [18]), it can be shown that any weak solution for $u, v$, and $w$ satisfies an energy inequality similar to the one for $\left\{u_{N}, v_{N}, w_{N}\right\}$. Hence, $u, v$, and $w$ satisfy the same bounds as $u_{N}, v_{N}$, and $w_{N}$. Consequently, by the linearity of the system $f(\phi, \theta) \equiv 0$, we have $u=v=w=0$ and thus, the weak solution is unique.

\section{Existence of a Solution to the Auxiliary Problem}

As the auxiliary problem possesses a unique weak solution for each continuous $q(t)$, we can now define a mapping $\mathscr{F}$ : $C([0, T]) \rightarrow C([0, T])$ via

$$
(\mathscr{F} q)(t)=\int_{\Omega} w(\phi, \theta, t ; q) \mathrm{d} \Omega .
$$

First, we show that $(\mathscr{F} q)(t)$ is bounded and continuous on $[0, T]$. From integrating the pointwise representation for $w(\phi, \theta, t ; q)$, we see that

$$
\int_{\Omega} w \mathrm{~d} \Omega=\beta \int_{\Omega} \int_{0}^{t} v e^{-\gamma(t-\tau)} \mathrm{d} \tau \mathrm{d} \Omega
$$

and, taking into account the fact that $0<e^{-\gamma(t-\tau)} \leq 1$, we have

$$
\begin{aligned}
\int_{\Omega} w \mathrm{~d} \Omega \leq & \beta \int_{0}^{t} \int_{\Omega}|v| \mathrm{d} \Omega \mathrm{d} \tau \\
= & \beta\left|\int_{0}^{t} \int_{\Omega}\right| v|\sin \phi \mathrm{d} \theta \mathrm{d} \phi \mathrm{d} \tau| \\
\leq & \beta\left(\int_{0}^{t} \int_{\Omega} \sin \phi \mathrm{d} \theta \mathrm{d} \phi \mathrm{d} \tau\right)^{1 / 2} \\
& \times\left(\int_{0}^{t} \int_{\Omega} v^{2} \sin \phi \mathrm{d} \theta \mathrm{d} \phi \mathrm{d} \tau\right)^{1 / 2} \\
\leq & \beta T^{1 / 2}[\operatorname{mes}(\Omega)]^{1 / 2}\left(\int_{Q_{T}} v^{2} \sin \phi \mathrm{d} Q_{T}\right)^{1 / 2} \\
\leq & C_{2},
\end{aligned}
$$

via the Schwartz inequality, where $C_{2}$ is a positive constant that depends on $C_{1}$ of Section 3, $T$, and mes $(\Omega)$.

For the continuity of $(\mathscr{F} q)(t)$, we consider $0 \leq t_{1}<t_{2} \leq$ $T$, and thus

$$
\begin{aligned}
& \left|\int_{\Omega} w\left(\phi, \theta, t_{1} ; q\right) \mathrm{d} \Omega-\int_{\Omega} w\left(\phi, \theta, t_{2} ; q\right) \mathrm{d} \Omega\right| \\
& \leq \beta \int_{\Omega} \mid \int_{0}^{t_{1}} v(\phi, \theta, \tau ; q) e^{-\gamma\left(t_{1}-\tau\right)} \mathrm{d} \tau \\
& -\beta \int_{0}^{t_{2}} v(\phi, \theta, \tau ; q) e^{-\gamma\left(t_{2}-\tau\right)} \mathrm{d} \tau \mid \mathrm{d} \Omega
\end{aligned}
$$

$$
\begin{aligned}
\leq & \beta \int_{\Omega}\left|\int_{t_{1}}^{t_{2}} v e^{-\gamma\left(t_{2}-\tau\right)} \mathrm{d} \tau\right| \mathrm{d} \Omega \\
& +\beta \int_{\Omega}\left|\int_{0}^{t_{1}} v\left[e^{-\gamma\left(t_{1}-\tau\right)}-e^{-\gamma\left(t_{2}-\tau\right)}\right] \mathrm{d} \tau\right| \mathrm{d} \Omega \\
\leq & \beta \int_{\Omega} \int_{t_{1}}^{t_{2}}|v| \mathrm{d} \tau \mathrm{d} \Omega \\
& +\beta \int_{\Omega} \int_{0}^{t_{1}}|v| e^{-\gamma\left(t_{1}-\tau\right)}\left[1-e^{\gamma\left(t_{1}-\tau\right)-\gamma\left(t_{2}-\tau\right)}\right] \mathrm{d} \tau \mathrm{d} \Omega \\
\leq & \beta \int_{\Omega} \int_{t_{1}}^{t_{2}}|v| \mathrm{d} \tau \mathrm{d} \Omega \\
& +\beta \int_{\Omega} \int_{0}^{t_{1}}|v|\left[1-e^{-\gamma\left(t_{2}-t_{1}\right)}\right] \mathrm{d} \tau \mathrm{d} \Omega \\
\leq & \beta \int_{\Omega} \int_{t_{1}}^{t_{2}}|v| \mathrm{d} \tau \mathrm{d} \Omega \\
& +\beta \int_{\Omega} \int_{0}^{t_{1}}|v| \gamma\left(t_{2}-t_{1}\right) \mathrm{d} \tau \mathrm{d} \Omega \\
\leq & C_{3}\left(t_{2}-t_{1}\right)^{1 / 2}+C_{4}\left|t_{2}-t_{1}\right|,
\end{aligned}
$$

where we used the fact that $0<e^{-\gamma(t-\tau)} \leq 1$, estimated $1-e^{-\gamma\left(t_{2}-t_{1}\right)}$ via the mean value theorem, and estimated the last two integrals exactly as the integral in (48). We note that $C_{3}$ and $C_{4}$ have the same dependence as $C_{2}$, which is independent of $q$ since $C_{1}$ is independent of $q$.

It therefore follows from (48) and (49) that $\mathscr{F}$ maps $C([0, T])$ into a convex subset of itself. All that remains for an application of Schauder's fixed point theorem is to show that $\mathscr{F}$ is continuous in the norm $\|\cdot\|_{\infty}$ of the separable Banach space $\mathscr{B}\left(C([0, T]),\|\cdot\|_{\infty}\right)$.

To show that $(\mathscr{F} q)(t)$ is continuous in $\|\cdot\|_{\infty}$, we consider

$$
\begin{aligned}
& \left(\mathscr{F} q_{1}\right)(t)-\left(\mathscr{F} q_{2}\right)(t) \\
& \quad=\beta \int_{\Omega} \int_{0}^{t}\left[v\left(\phi, \theta, \tau ; q_{1}\right)-v\left(\phi, \theta, \tau ; q_{2}\right)\right] e^{-\gamma(t-\tau)} \mathrm{d} \tau \mathrm{d} \Omega,
\end{aligned}
$$

where $v\left(\phi, \theta, \tau ; q_{i}\right)$ is the weak solution of (20) for continuous $q_{i}(t), 0 \leq t \leq T$, for $i=1,2$. Forming the difference of (20) for $q_{i}(t)$, for $i=1,2$, we have

$$
\begin{aligned}
-\int_{\mathrm{Q}_{T}}\left(u_{1}-u_{2}\right) g_{t} \sin \phi \mathrm{d} Q_{T}+\int_{\mathrm{Q}_{T}}\left(u_{1 \phi}-u_{2 \phi}\right) g_{\phi} \sin \phi \mathrm{d} Q_{T} \\
\quad+\int_{\mathrm{Q}_{T}}\left(u_{1 \theta}-u_{2 \theta}\right) g_{\theta} \csc \phi \mathrm{d} Q_{T} \\
=-\int_{\mathrm{Q}_{T}}\left[\alpha\left(q_{1}\right) u_{1}-\alpha\left(q_{2}\right) u_{2}\right] g \sin \phi \mathrm{d} Q_{T} \\
\quad+\gamma \int_{Q_{T}}\left(w_{1}-w_{2}\right) g \sin \phi \mathrm{d} Q_{T}, \quad \forall g \in W^{1,1},
\end{aligned}
$$




$$
\begin{aligned}
-\int_{Q_{T}} & \left(v_{1}-v_{2}\right) g_{t} \sin \phi \mathrm{d}_{T}+\int_{Q_{T}}\left(v_{1 \phi}-v_{2 \phi}\right) g_{\phi} \sin \phi \mathrm{d}_{T} \\
& +\int_{\mathrm{Q}_{T}}\left(v_{1 \theta}-v_{2 \theta}\right) g_{\theta} \csc \phi \mathrm{d}_{T} \\
= & \int_{\mathrm{Q}_{T}}\left[\alpha\left(q_{1}\right) u_{1}-\alpha\left(q_{2}\right) u_{2}\right] g \sin \phi \mathrm{dQ}_{T} \\
& -\beta \int_{\mathrm{Q}_{T}}\left(v_{1}-v_{2}\right) g \sin \phi \mathrm{d} \mathrm{Q}_{T}, \quad \forall g \in W^{1,1},
\end{aligned}
$$

$$
\begin{aligned}
& w_{1}\left(\phi, \theta, t ; q_{1}\right)-w_{2}\left(\phi, \theta, t ; q_{2}\right) \\
& \quad=\beta \int_{0}^{t}\left[v_{1}\left(\phi, \theta, t ; q_{1}\right)-v_{2}\left(\phi, \theta, t ; q_{2}\right)\right] e^{-\gamma(t-\tau)} \mathrm{d} \tau,
\end{aligned}
$$

where the $u_{i}, v_{i}$, and $w_{i}$ depend upon the $q_{i}$, for $i=1,2$, as indicated by the $w_{i}$ and $v_{i}$ expressed in (52). Also, in (51) we can write

$$
\begin{aligned}
\int_{Q_{T}}[\alpha & \left.\left(q_{1}\right) u_{1}-\alpha\left(q_{2}\right) u_{2}\right] g \sin \phi \mathrm{d}_{T} \\
= & \int_{Q_{T}}\left[\alpha\left(q_{1}\right)-\alpha\left(q_{2}\right)\right] u_{1} g \sin \phi \mathrm{d}_{T} \\
& \quad+\int_{Q_{T}} \alpha\left(q_{2}\right)\left[u_{1}-u_{2}\right] g \sin \phi \mathrm{d}_{T} .
\end{aligned}
$$

Hence, if the $u, v, w$, and $\alpha(q)$ as in (20) were, respectively, replaced by $u_{1}-u_{2}, v_{1}-v_{2}, w_{1}-w_{2}$, and $\alpha\left(q_{2}\right)$ above, and if the term $\int_{D_{0}} g f \sin \phi \mathrm{d} D_{0}$ was deleted, then the right-hand side of (52) would only contain the term

$$
\int_{\mathrm{Q}_{T}}\left[\alpha\left(q_{1}\right)-\alpha\left(q_{2}\right)\right] u_{1} g \sin \phi \mathrm{d} Q_{T}
$$

to be estimated, instead of the initial data term $\int_{D_{0}} g f \sin \phi \mathrm{d} D_{0}$. Now the term (55) can be estimated as follows:

$$
\begin{aligned}
\left|\int_{Q_{T}}\left[\alpha\left(q_{1}\right)-\alpha\left(q_{2}\right)\right] u_{1} g \sin \phi \mathrm{d} Q_{T}\right| \\
\leq\left[\int_{Q_{T}}\left[\alpha\left(q_{1}(\tau)\right)-\alpha\left(q_{2}(\tau)\right)\right]^{2} u_{1}^{2} \sin \phi \mathrm{d} Q_{T}\right]^{1 / 2} \\
\quad \times\left[\int_{Q_{T}} g^{2} \sin \phi \mathrm{d} Q_{T}\right]^{1 / 2} \\
\leq \frac{1}{2} \int_{Q_{T}}\left[\alpha\left(q_{1}(\tau)\right)-\alpha\left(q_{2}(\tau)\right)\right]^{2} u_{1}^{2} \sin \phi \mathrm{d} Q_{T} \\
\quad+\frac{1}{2} \int_{Q_{T}} g^{2} \sin \phi \mathrm{d} Q_{T} \\
\leq \frac{1}{2}\left(\|\alpha\|_{\infty}+\|\alpha\|_{\infty}\right)\left\|\frac{d \alpha}{d q}\right\|_{\infty}\left\|q_{1}-q_{2}\right\|_{\infty}
\end{aligned}
$$

$$
\begin{aligned}
& \times \int_{Q_{T}} u_{1}^{2} \sin \phi \mathrm{d} Q_{T}+\frac{1}{2} \int_{\mathrm{Q}_{T}} g^{2} \sin \phi \mathrm{d}_{T} \\
\leq & C_{5}\left\|q_{1}-q_{2}\right\|_{\infty}+\frac{1}{2} \int_{Q_{T}} g^{2} \sin \phi \mathrm{d} Q_{T},
\end{aligned}
$$

where $C_{5}$ depends on the bound for $\left\|u_{1}\right\|_{Q_{T}}$ which is given by (39) for the weak solution of (20) and is independent of $q_{1}$. The second term on the right-hand side of (54) and (56) involving the $g$ becomes $u_{1}-u_{2}$ in the energy inequality associated with (52). We now have a replay of (37), (38), and (39), with $\|f\|_{D_{0}}^{2}$ replaced with $C_{5}\left\|q_{1}-q_{2}\right\|_{\infty}$. From the estimate of $\left\|v_{1}-v_{2}\right\|_{Q_{T}}^{2}$ resulting from the Galerkin estimate for $\left\|u_{1}-u_{2}\right\|_{\mathrm{Q}_{T}}^{2}+\left\|v_{1}-v_{2}\right\|_{\mathrm{Q}_{T}}^{2}+\left\|w_{1}-w_{2}\right\|_{\mathrm{Q}_{T}}^{2}$ in terms of $C_{6}\left\|q_{1}-q_{2}\right\|_{\infty}$, we obtain the estimation of (50) below:

$$
\left\|\mathscr{F}\left(q_{1}\right)-\mathscr{F}\left(q_{2}\right)\right\|_{\infty} \leq C_{7}\left\|q_{1}-q_{2}\right\|_{\infty} .
$$

This leads to the following statement.

Theorem 1. From (57), one infers the continuity of the mapping $\mathscr{F}$ and the validity of the application of the Schauder fixed point theorem, yielding the existence of a weak solution of the weak formulation of (7), (8), and (9), given by (16), (18), and (19).

Proof. See the preceding analysis.

\section{Future Considerations}

We leave open for consideration the establishment of a unique solution to the weak formulation developed in this paper. This will entail a numerous amount of estimates, which are too tedious to be included in the present work. We refer the interested reader to Chapter 3, Section 2, of [18], as we anticipate that analogues of the energy inequalities derived therein can be generalized to ones that apply to our current problem. We also anticipate that these inequalities will ultimately produce a contraction mapping via a bootstrapping procedure. The constants in these inequalities will have to be estimated closely enough to show the dependence on (small) $t$, in order to permit the construction of the aforementioned contraction mapping.

\section{Conflict of Interests}

The authors declare that there is no conflict of interests regarding the publication of this paper.

\section{Acknowledgments}

The authors thank the referee for the timely insights, suggestions, and inquiries. In addition, they appreciate the assistance of Frank B. Jones (Rice University) for sharing with them his unpublished lecture notes pertaining to the heat equation on the unit sphere. 


\section{References}

[1] J. R. Cannon and D. J. Galiffa, "A numerical method for a nonlocal elliptic boundary value problem," Journal of Integral Equations and Applications, vol. 20, no. 2, pp. 243-261, 2008.

[2] F. J. S. A. Corrêa and C. de Morais Filho, "On a class of nonlocal elliptic problems via Galerkin method," Journal of Mathematical Analysis and Applications, vol. 310, pp. 177-187, 2005.

[3] J. R. Cannon and D. J. Galiffa, "On a numerical method for a homogeneous, nonlinear, nonlocal, elliptic boundary value problem," Nonlinear Analysis: Theory, Methods \& Applications, vol. 74, no. 5, pp. 1702-1713, 2011.

[4] J. R. Cannon and D. J. Galiffa, "An epidemiology model suggested by yellow fever," Mathematical Methods in the Applied Sciences, vol. 35, no. 2, pp. 196-206, 2012.

[5] D. J. Galiffa and J. R. Cannon, "Nonlocal modeling of insect borne diseases," in Fevers: Types, Treatments and Health Risks, chapter 8, Nova Science, New York, NY, USA, 2013.

[6] V. Capasso and R. E. Wilson, "Analysis of a reaction-diffusion system modeling man-environment-man epidemics," SIAM Journal on Applied Mathematics, vol. 57, no. 2, pp. 327-346, 1997.

[7] F. van den Bosch, J. A. J. Metz, and O. Diekmann, "The velocity of spatial population expansion," Journal of Mathematical Biology, vol. 28, no. 5, pp. 529-565, 1990.

[8] W. Wang, Y. Cai, M. Wu, K. Wang, and Z. Li, "Complex dynamics of a reaction-diffusion epidemic model," Nonlinear Analysis: Real World Applications, vol. 13, no. 5, pp. 2240-2258, 2012.

[9] W. Wang and X.-Q. Zhao, "Basic reproduction numbers for reaction-diffusion epidemic models," SIAM Journal on Applied Dynamical Systems, vol. 11, no. 4, pp. 1652-1673, 2012.

[10] M. E. Alexander, S. M. Moghadas, G. Röst, and J. Wu, "A delay differential model for pandemic influenza with antiviral treatment," Bulletin of Mathematical Biology, vol. 70, no. 2, pp. 382-397, 2008.

[11] A. Lunelli, A. Pugliese, and C. Rizzo, "Epidemic patch models applied to pandemic influenza: contact matrix, stochasticity, robustness of predictions," Mathematical Biosciences, vol. 220, no. 1, pp. 24-33, 2009.

[12] R. Patel, I. M. Longini Jr., and M. E. Halloran, "Finding optimal vaccination strategies for pandemic influenza using genetic algorithms," Journal of Theoretical Biology, vol. 234, no. 2, pp. 201-212, 2005.

[13] G. Chowell, C. E. Ammon, N. W. Hengartner, and J. M. Hyman, "Transmission dynamics of the great influenza pandemic of 1918 in Geneva, Switzerland: assessing the effects of hypothetical interventions," Journal of Theoretical Biology, vol. 241, no. 2, pp. 193-204, 2006.

[14] L. Rass, "Pandemic bounds for an epidemic on an infinite lattice," Mathematical Biosciences, vol. 195, no. 2, pp. 194-209, 2005.

[15] N. I. Akinwande, "A mathematical model of the dynamics of the HIV/AIDS disease pandemic," Journal of the Nigerian Mathematical Society, vol. 25, pp. 99-108, 2006.

[16] C. Castillo-Chavez and G. Chowell-Puente, "Special issue on mathematical models, challenges, and lessons learned from the 2009 A/H1N1 influenza pandemic," Mathematical Biosciences and Engineering, vol. 8, pp. 1-6, 2011.

[17] W. Jumpen, B. Wiwatanapataphee, Y. H. Wu, and I. M. Tang, "A SEIQR model for pandemic influenza and its parameter identification," International Journal of Pure and Applied Mathematics, vol. 52, no. 2, pp. 247-265, 2009.
[18] O. A. Ladyženskaja, V. A. Solonnikov, and N. N. Ural'ceva, Linear and Quasi-Linear Equations of Parabolic Type, American Mathematical Society, 1968. 


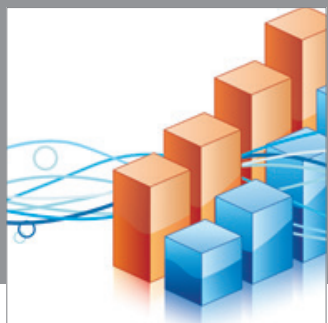

Advances in

Operations Research

mansans

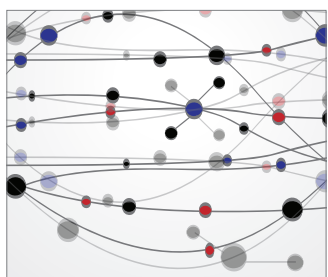

The Scientific World Journal

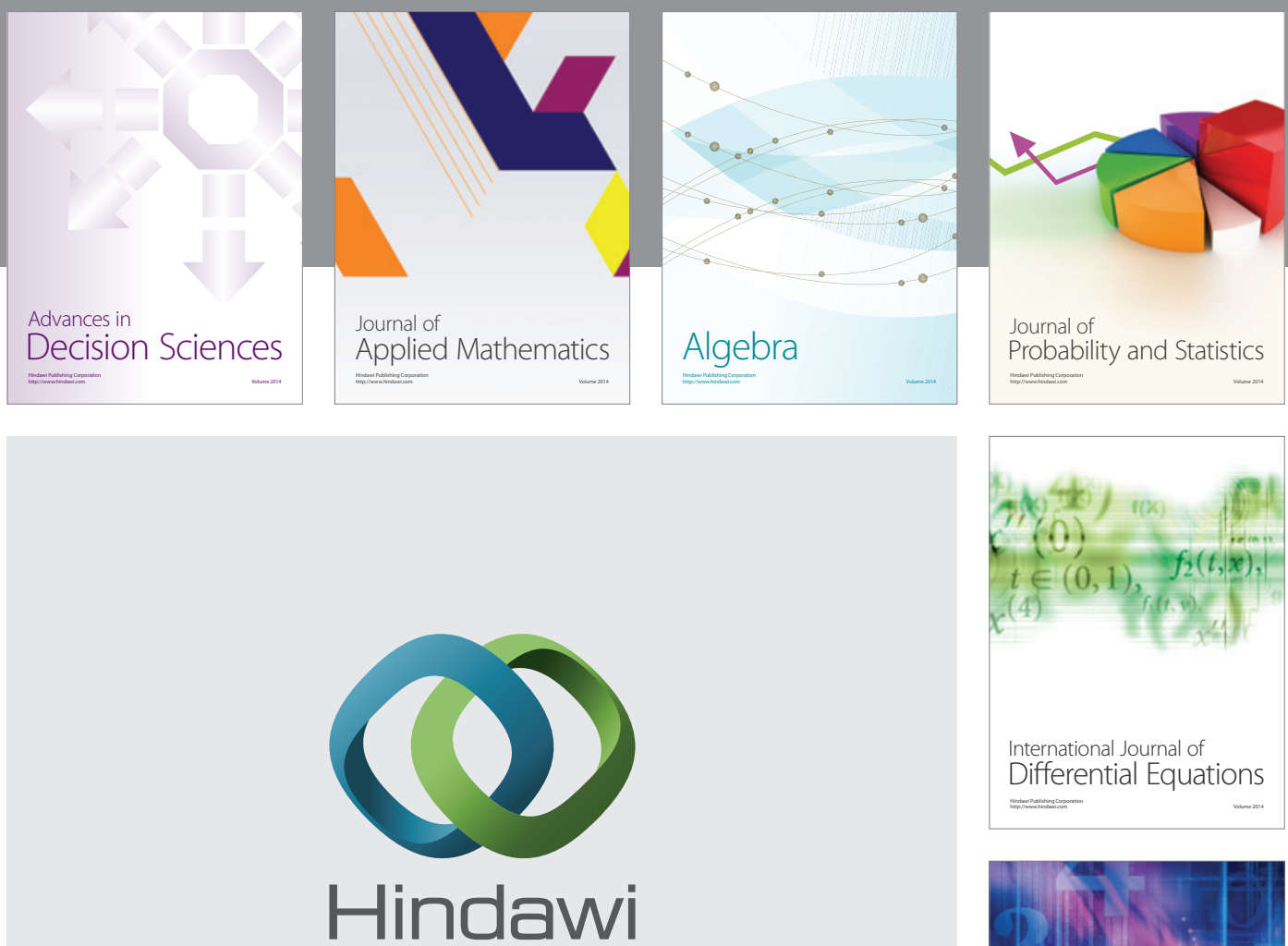

Submit your manuscripts at http://www.hindawi.com
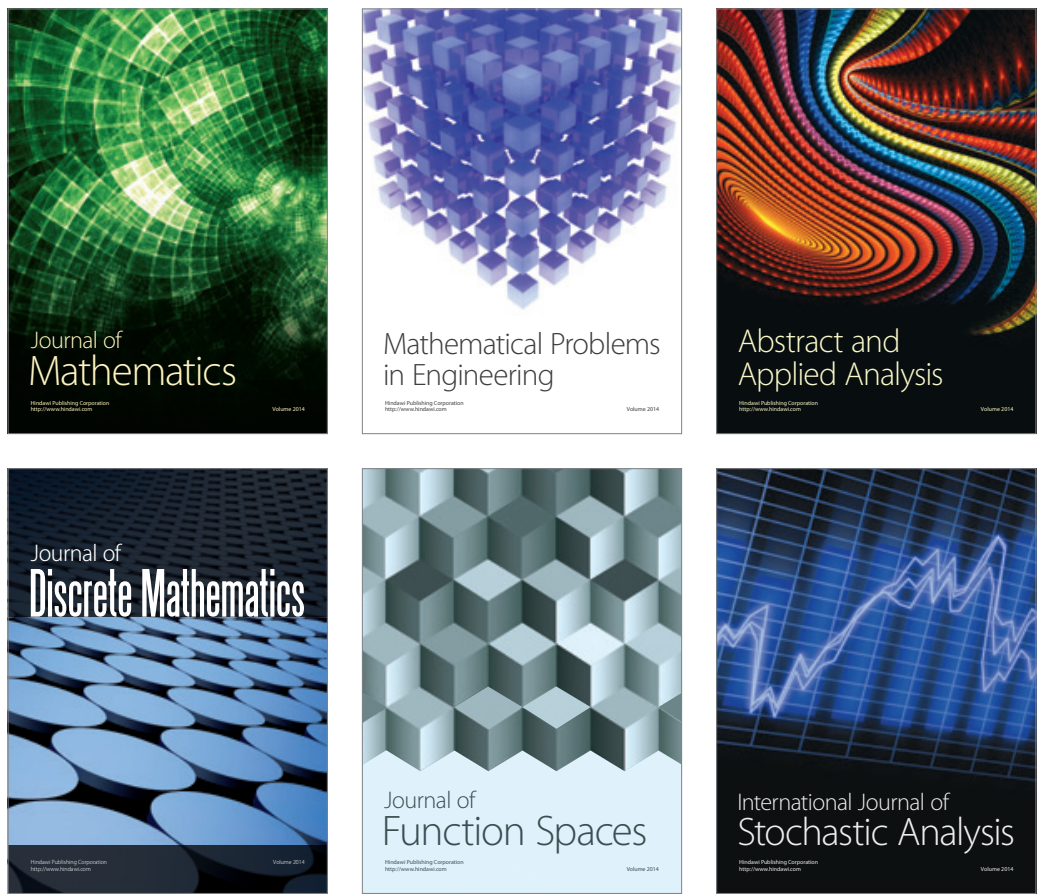

Journal of

Function Spaces

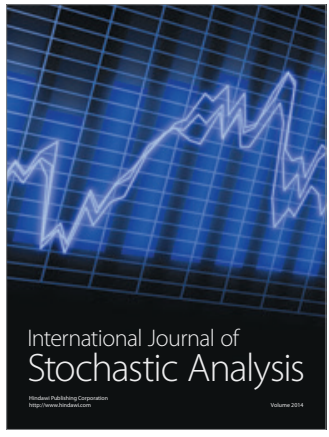


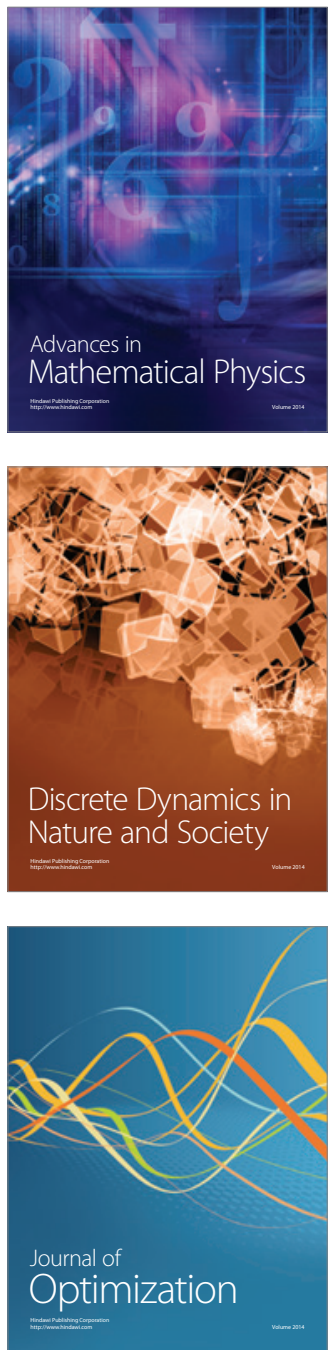